\title{
Strict Identity, Coherence, and Parallelism in VP Ellipsis
}

\author{
Christina S. Kim and Jeffrey T. Runner \\ University of Rochester
}

\section{Background}

In sentences containing VP ellipsis, the understood meaning of the elided phrase is similar to that of an overt phrase elsewhere in the sentence (the antecedent). In particular, the elided material can't be construed as something else; for example, (1) is understood as true only if Bill reported the problem.

\section{(1) John reported the problem, and Bill did too.}

The exact nature of the similarity that must obtain between antecedent and ellipsis site has been under debate. Intuitively, it is a condition associated with recoverability: when a VP-gap is encountered, a VP representation from the preceding context must be interpreted in the empty VP position ${ }^{1}$. Syntactic identity has been argued for based on sentences where syntactic non-identity results in illformedness (Sag 1976, Williams 1977). For example, in sentences like (2a) (Kehler 2000), the antecedent and elided VP differ in Voice, violating syntactic identity. On the assumption that syntactic identity requires identity of binding relations, the identity preserving interpretation of ( $2 b$ ) is the so-called sloppy reading, where John blamed John, and Bill blamed Bill. The indexation in (2b) (Kitagawa 1991), which reflects the strict reading (where both John and Bill blamed John), is ruled out, presumably by Condition A.

(2) a. *This problem was looked into by John, and Bill did too.

b. $* J_{o h n}$ blamed himself $_{i}$, and Bill did too [blame $\mathrm{x}_{i}$ ].

(3) a. ?This problem was looked into by John, even though Bill already had.

b. $\mathrm{John}_{i}$ blamed himself $_{i}$, because Bill did [blame $\mathrm{x}_{i}$ ].

However, sentences like (3) seem to improve in acceptability, despite being superficially analogous to those in (2). Such data has been used to motivate a semantic identity condition on VP ellipsis, to rule in acceptable sentences containing some form of syntactic non-identity (Dalrymple et al. 1991).

Here, we focus on two proposals designed to capture the kinds of contrasts in (2) and (3), which differ in the level of representation that VP ellipsis resolution is assumed to take place.

We thank Stephanie Huston and Kim Leiken for help collecting data. This work was supported by NSF grant BCS-0518842.

${ }^{1}$ The notion of recoverability of deletion goes back at least as far as Chomsky (1965). 
According to Kehler (2000, 2002), VP ellipsis is resolved at a level of discourse representation that encodes coherence relations-Kehler suggests that the difference between (2) versus (3) is due to different discourse coherence relations that hold between antecedent and ellipsis clauses ${ }^{2}$. The apparent paradox of certain sentences strictly adhering to syntactic identity and other sentences permitting violations of syntactic identity arises because both syntactic and semantic mechanisms for VP recovery are available. Which one applies is determined by the coherence relation between the clauses containing the antecedent and elided VPs. A Resemblance relation-in which two clauses are typically being compared and parallelism is highlighted - requires aligning syntactic arguments between two clauses. As such, VP ellipsis resolution should have to recover a syntactic antecedent (4a), and sensitivity to syntactic identity — such as Voice features-and Condition A of the Binding Theory might be expected. Establishing a Cause-Effect relation, on the other hand, only requires relating the propositional meanings of the two clauses. In such cases, VP ellipsis is resolved by recovering a higher-order semantic antecedent (4b), explaining the insensitivity to syntactic identity or Condition A.

(4)

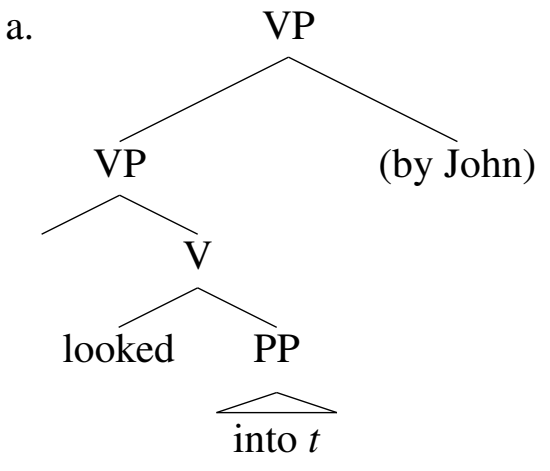

b. $\lambda x . l o o k-i n t o(x$, problem $)$

In contrast, Hestvik (1995) attributes the contrast between (2b) and (3b) to a structural difference: while (3b) arguably involves symmetric coordination (5a), in $(2 b)$, the ellipsis is in a subordinate clause $(5 b)^{3}$.

(5)

a.

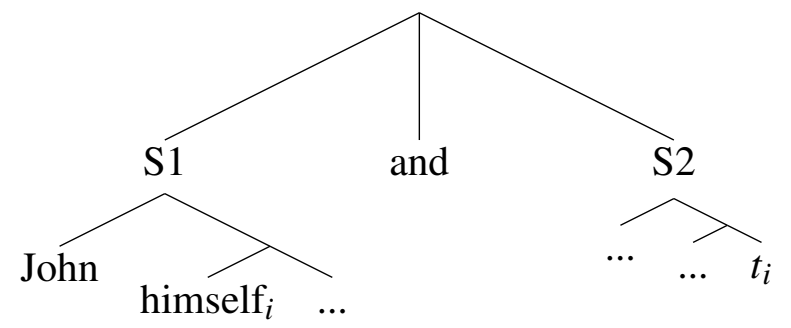

\footnotetext{
${ }^{2}$ For similar proposals, see Hobbs (1979), Asher (1993), Prust et al. (1994), Asher et al. (2001)

${ }^{3}$ Whether coordination is syntactically symmetric or asymmetric has been a controversial question; see e.g. discussions in Gazdar et al. (1985), Johannessen (1993), Kayne (1994), Munn (2000).
} 
b.

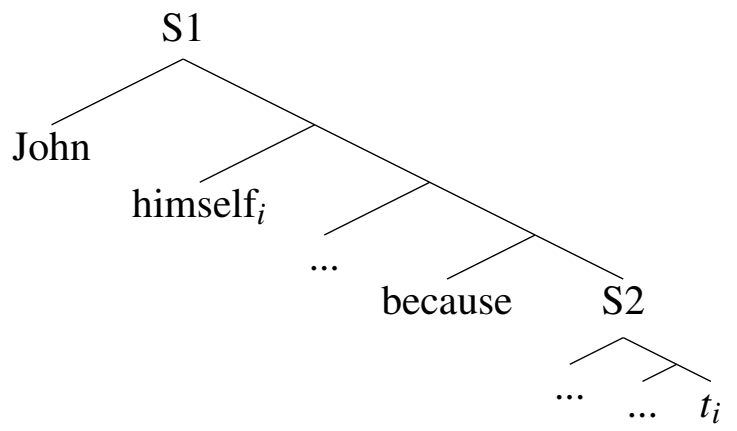

Hestvik assumes an analysis that links antecedents and anaphors derivationally by adjoining the reflexive to its antecedent at LF (Lebeaux 1986, Chomsky 1986, Katada 1991). The asymmetry between the coordination and subordination cases results from the interaction between copying the antecedent VP and LF raising of the reflexive. To derive a sloppy reading from (6a), the antecedent VP is copied into the ellipsis site first, yielding an intermediate representation like (6b); then the two reflexives undergo LF movement, as in (6c).

(6) a. [ ${ }_{S 1}$ John blamed himself $] \ldots\left[{ }_{S 2}\right.$ Bill did $]$

b. [ $s_{1}$ John $\left[V_{P}\right.$ blamed himself $\left.\left.f_{i}\right]\right] \ldots$ [s2 Bill [vP blamed himself $\left.\left.j\right]\right]$

c. $\left[s 1\right.$ John $\left[\alpha\right.$ himself $_{i}\left[{ }_{V P}\right.$ blamed $\left.\left.\left.t_{i}\right]\right]\right] \ldots\left[S 2\right.$ Bill $\left[\alpha\right.$ himself $_{j}[v P$ blamed $\left.\left.\left.t_{j}\right]\right]\right]$

Because each instance of 'himself' is bound by its local subject, this derivation goes through no matter how S1 and S2 are related structurally; thus both coordination and subordination structures allow sloppy identity interpretations.

Deriving a strict identity reading requires ordering reflexive movement before VP copying. Because the antecedent VP in the intermediate representation (7a) has an indexed trace after reflexive movement, it is this VP that gets copied into the ellipsis site in (7b).

(7) a. [S1 John $\left[\alpha \operatorname{himself}_{i}\left[V_{P}\right.\right.$ blamed $\left.\left.\left.t_{i}\right]\right]\right] \ldots\left[S_{2}\right.$ Bill $\left.\left[V_{P} e\right]\right]$

b. $\left[s_{1}\right.$ John $\left[\alpha\right.$ himself $_{i}\left[V_{P}\right.$ blamed $\left.\left.\left.t_{i}\right]\right]\right] \ldots\left[{ }_{S 2}\right.$ Bill [VP blamed $\left.\left.t_{i}\right]\right]$

Now that both VPs contain traces that must be bound by a single reflexive at LF, it matters how the moved reflexive, in S1, is structurally related to the elided VP, in S2. Sentences like (2b) and (3b) differ in the ability of the first NP 'John' to asymmetrically c-command the variable in the ellipsis site; as a consequence, the subject of the first clause can bind the variable in the ellipsis clause for (3b), but not (2b). While the details of the analysis are not crucial here, what is important for our purposes is that VP ellipsis is assumed to be resolved at a level of syntactic structure that encodes hierarchical relations like c-command.

These proposals make different predictions about the environments in which deviations from strict identity will result in degraded acceptability. On a coherencebased account, strict identity readings of sentences like (3b) should be restricted to those cases where the antecedent and ellipsis clauses are related by a Cause-Effect relation. Further, since relations like Resemblance and Cause-Effect are a feature 
of discourse structure rather than syntactic structure, the pattern of acceptability should be independent of whether the elided VP and its antecedent are in the same sentence or different sentences. However, since the structural c-command relation is central to the structural account of sentences like (3b), strict identity readings should be restricted to within-sentence VP ellipsis, where both VPs are part of a connected syntactic structure. On the other hand, a structural account like Hestvik's offers no obvious explanation of the acceptability contrast between (2a) and (3a), which involve violations of syntactic identity but no variable binding, whereas a coherence-based account predicts that Voice mismatch and strict identity should be possible under the same discourse coherence conditions, in both sentence-internal and cross-sentential ellipsis.

We present the results of three experiments that test these predictions. Experiment 1 compares sentences containing reflexives, where ellipsis is either across two sentences or contained in a single sentence. Consistent with the structural account, we find that strict readings are limited to subordination structures, and only to sentence-internal ellipsis. While these results provided no evidence for a coherence-based explanation that does not distinguish relations between structural representations within and across sentences, Experiments 2 and 3 confirm the predictions of the coherence account: degradation in acceptability due to syntactic identity violations is modulated by discourse coherence, with no difference between sentence-internal and cross-sentential ellipsis. Any unified account of VP ellipsis resolution will have to explain these different patterns of data for reflexive binding and Voice mismatch, particularly with respect to the nature of the representations that are subject to the identity condition on ellipsis. In the Discussion section, we outline some questions that this work gives rise to, and some methodological directions for pursuing them.

\section{Experiment 1: Reflexives and Strict Identity}

Experiment 1 looked at sentences with VP ellipsis containing reflexives (2b, 3b), manipulating two factors: the discourse relation between the antecedent and ellipsis clauses (Resemblance or Cause-Effect), and the ellipsis type-whether the antecedent-ellipsis relation was within the same sentence (within-sentence), or spanned two sentences (cross-sentence). This resulted in four experimental conditions, exemplified in (8).

(8) a. Within-sentence, Resemblance: Jane voted for herself in the election, and Ann did too.

b. Within-sentence, Cause-Effect: Jane voted for herself in the election, so Ann did too.

c. Cross-sentence, Resemblance: Jane voted for herself in the election. Ann did too.

d. Cross-sentence, Cause-Effect: Jane voted for herself in the election. So Ann did too. 
After participants read a sentence or sentence pair in one of the four conditions in (8), they answered a two-choice question like (9), designed to reveal how they had interpreted the sentence.

(9) Who did Ann vote for?
(A) Ann
(B) Jane

In the current example, choosing (A) Ann indicates a sloppy, or bound-variable reading, while choosing (B) Jane indicates a strict, or coreferential reading. Both the coherence-based and structural accounts predict that more strict interpretations than sloppy interpretations should be observed in the within-sentence cases. However only the coherence account predicts that this asymmetry should extend to crosssentential ellipsis.

18 University of Rochester students who were native speakers of English participated in the experiment. The dependent measure was the proportion of strict identity interpretations observed in each cell of the experiment. A mixed-effects logit model was fit to the proportion data, with Subject as a random effect (Gelman and Hill 2007, Baayen 2008).

\subsection{Results}

The proportions of responses indicating strict interpretations are shown in Figure 1. There were main effects of both ellipsis type and discourse relation: there were more strict interpretations within sentences than across sentences, and more strict interpretations were observed when clauses were related by Cause-Effect than when they were related by Resemblance.

Importantly, there was an interaction between ellipsis type and discourse relation, such that the proportion of strict interpretations was greater for CauseEffect than Resemblance, in within- but not cross-sentence ellipsis ${ }^{4}$.

This pattern of results is consistent with the structural account, but remains unexplained in a coherence-based account. Since coherence relations are relations between clause meanings, they should hold equally between conjoined clauses and pairs of sentences. Specifically, Kehler's explanation of apparent syntactic effects in VP ellipsis is that retrieving the structure of the antecedent is a byproduct of establishing a Resemblance relation, which involves comparing the arguments of one clause to the corresponding arguments in the other clause. Explaining the contrast between ( $2 b$ ) and (3b) on the basis of coherence relies on the assumption that sloppy identity satisfies syntactic parallelism while strict identity violates it-no notion of c-command need be invoked. However, it is precisely the lack of this syntactic relation in cross-sentential VP ellipsis that distinguishes cases where strict readings seem to be prohibited in the experimental data.

\footnotetext{
${ }^{4}$ Interestingly, there is a surprisingly high rate of strict identity responses across the board (greater than .35 in all conditions). We set this aside here, but note that it is unexpected from the point of view of Condition A.
} 


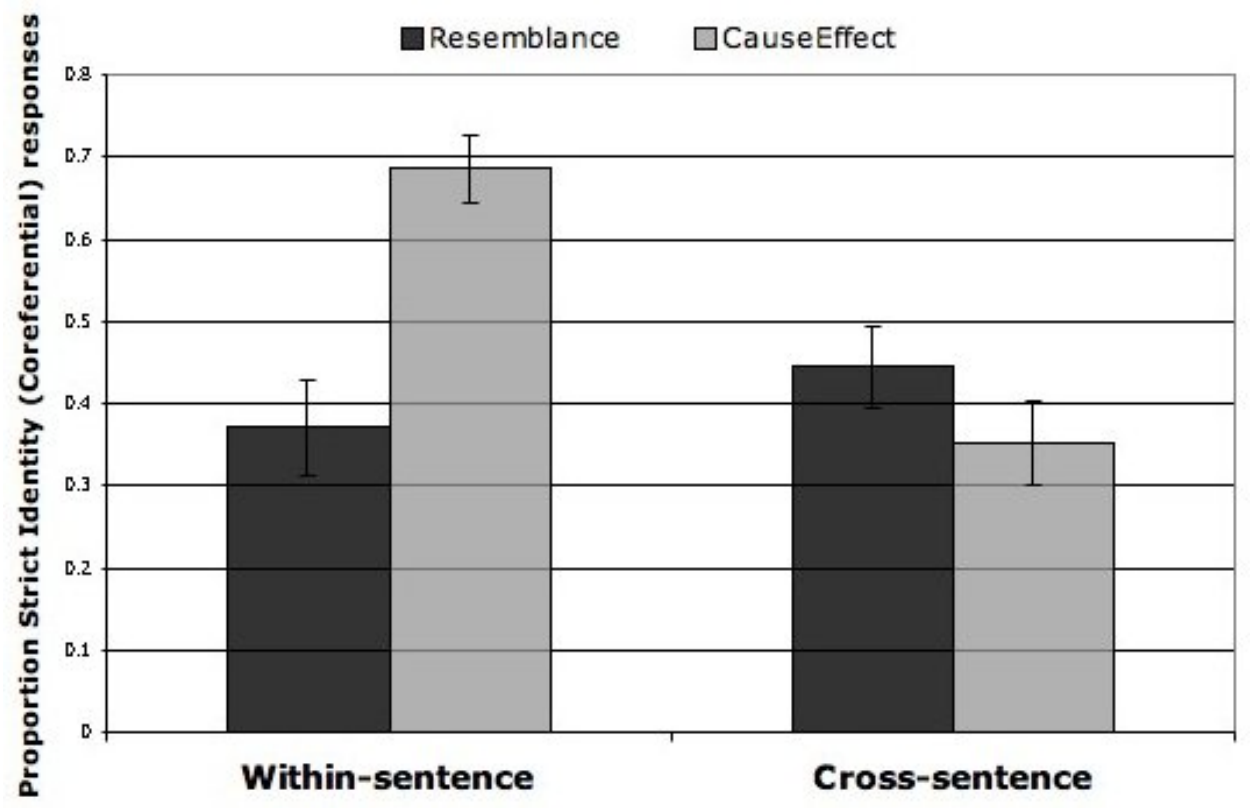

Figure 1: Experiment 1-Mean proportion of strict identity readings by condition (error bars represent Standard Error).

If we accept a Hestvik-style explanation of strict readings of reflexives in VP ellipsis, a problem that remains is how to account for the variable acceptability in other cases of structural mismatch, like the Voice mismatches in (2a) and (3a). In fact, previous work by Frazier and Clifton (2006) has argued against a coherence account of parallelism effects in VP ellipsis based on sentences with Voice mismatches, and the availability of strict and sloppy identity readings of reflexives. In other work, Frazier and Clifton (2005) have proposed that VP ellipsis is governed by different sets of constraints when it is sentence-internal (the syntactic domain) than when it spans a sentence boundary (the discourse domain): a syntactic antecedent is recovered only in the sentence-internal case, because detailed syntactic representations are assumed not to be available to the processor once a sentence has been closed off. If "syntactic" antecedents indeed have different representations from "discourse" antecedents, we might expect the pattern of acceptability in sentences like (2a) and (3a) to differ accordingly, producing the kind of interaction observed for reflexives in Experiment 1. Experiments 2 and 3 explore this possibility.

\section{Experiments 2-3: Voice Mismatches}

Are syntactic mismatch effects sensitive to the discourse coherence relation between antecedent and ellipsis clauses? In two magnitude estimation experiments (Bard et al. 1996), we compared the effect of Voice mismatch on the acceptability of VP ellipsis across discourse relations (Resemblance or Cause-Effect). Discourse 
relation was crossed with the presence/absence of ellipsis (Ellipsis or No Ellipsis), in Experiment 2, and with ellipsis type (within- or cross-sentence) in Experiment 3.

As in typical acceptability judgment studies, participants were asked to judge the acceptability of a series of sentences. Unlike in a fixed-scale rating study, participants assigned a score to each sentence relative to a standard score (or modulus) that they had chosen at the beginning of the experimental session. Thus participants established their own anchors for judging the sentences in the experiment.

Participants made proportional estimates relative to their modulus value. For example, if the modulus value chosen was 100 , a sentence that sounds twice as acceptable should be scored 200, while a sentence that sounds half as acceptable should be scored 50. Experiments 2 and 3 used the modulus sentence: "The kids were amused by the cartoon, but their parents weren't." On each trial, a sentence appeared on the screen along with the modulus sentence. Participants entered their score for the sentence in a text box.

All analyses were performed on log-transformed normalized scores. Log scores were fit to linear mixed-effects regression models, with Subject and Item as random effects.

\subsection{Experiment 2: Discourse Coherence Modulates Effects of Structural Mismatch}

The purpose of Experiment 2 was to confirm the contrast between sentences like (2a) and (3a) - that the degradation in sentences containing Voice mismatches is modulated by the discourse relation between the antecedent and ellipsis clauses. Sentences with (10) and without ellipsis (11) were compared across discourse relations (Resemblance or Cause-Effect). For each condition containing Voice mismatch, there was a corresponding condition where antecedent and ellipsis clauses matched in Voice, resulting in eight experimental conditions ${ }^{5}$.

a. Ellipsis, Resemblance:

Kurt blamed Mario for the terrible performance, and Jon $\{$ did, was $\}$ too.

b. Ellipsis, Cause-Effect:

Kurt blamed Mario for the terrible performance, so Jon $\{$ did, was $\}$ too.

a. No Ellipsis, Resemblance:

Kurt blamed Mario for the terrible performance, and Jon \{blamed him, was blamed for it $\}$ too.

b. No Ellipsis, Cause-Effect:

Kurt blamed Mario for the terrible performance, so Jon \{blamed him, was blamed for it $\}$ too.

For a coherence account, a syntactic antecedent is recovered in VP ellipsis resolution only when clauses are related by Resemblance-because interpreting

\footnotetext{
${ }^{5}$ All test items and fillers in the current experiment were single sentences. The following experiment includes both within-sentence and cross-sentential ellipsis.
} 
a sentence like (10b) does not involve accessing a syntactic representation of the antecedent VP, it should be insensitive to structural mismatches.

14 University of Rochester students who had not participated in Experiment 1 participated in the experiment.

\subsubsection{Results}

Mean log acceptability estimates for each of the conditions are shown in Figure 2. Focusing on the relevant contrasts, first, there was an interaction between syntactic mismatch and ellipsis: sentences containing Voice mismatches were judged less acceptable than their matched counterparts, but only when the second clause contained VP ellipsis. This merely confirms that the penalty associated with syntactic mismatch is a constraint specific to VP ellipsis, instead of reflecting a general preference for syntactic parallelism.

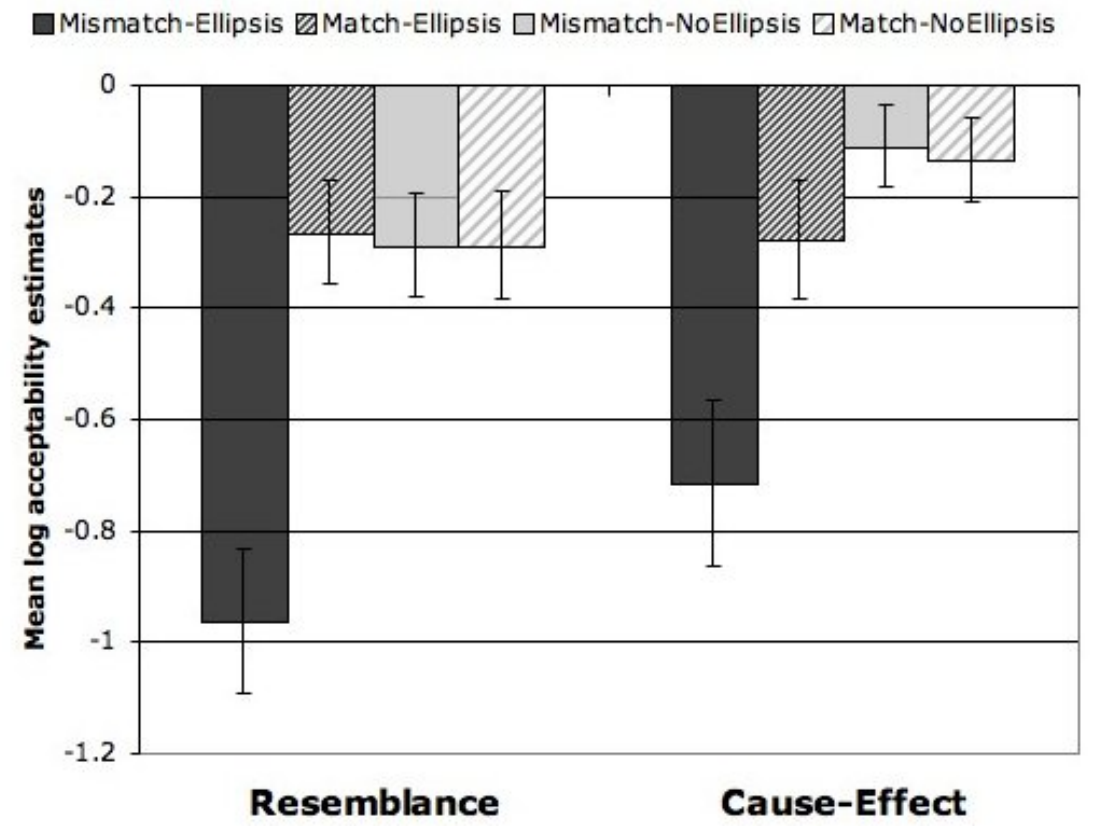

Figure 2: Experiment 2-Mean acceptability estimates (error bars represent Standard Error).

In addition, there was a three-way discourse relation-ellipsis-mismatch interaction, such that the ellipsis-related mismatch penalty was stronger when clauses were related by Resemblance, as opposed to Cause-Effect.

Consistent with the coherence account, the Cause-Effect relation alleviates the degradation associated with violating syntactic identity in VP ellipsis. However, the syntactic mismatch effects are not eliminated, and the fact that we observe gradient structural sensitivity suggests that syntactic structure must be recovered, even in cases where a strict coherence account would claim VP ellipsis is resolved via higher-order unification (Dalrymple et al. 1991). 
The results of Experiment 2 show that the coherence relations between antecedent and ellipsis clauses do influence how sentences with VP ellipsis are interpreted, in cases where an account based on structural asymmetry (Hestvik 1995) does not apply, at least in any obvious way. However, a major difference between the structural and coherence-based accounts is how cross-sentential VP ellipsis is expected to compare to sentence-internal ellipsis. Experiment 3 compares these cases directly.

\subsection{Experiment 3: Discourse Coherence Effects Within and Across Sentences}

Experiment 3 compared the acceptability of VP ellipsis within (12) and across sentences (13); as in Experiment 2, items varied in discourse relation (Resemblance or Cause-Effect), and whether the elided VP and its antecedent matched syntactically (Match or Mismatch). This resulted in eight experimental conditions.

a. Within-sentence, Resemblance:

Kurt blamed Mario for the terrible performance, and Jon \{did, was\} too.

b. Within-sentence, Cause-Effect:

Kurt blamed Mario for the terrible performance, so Jon $\{$ did, was $\}$ too.

a. Cross-sentence, Resemblance:

Kurt blamed Mario for the terrible performance. Jon $\{$ did, was $\}$ too.

b. Cross-sentence, Cause-Effect:

Kurt blamed Mario for the terrible performance. So Jon $\{$ did, was $\}$ too.

Based on Experiment 2, we expect Voice mismatch with a Resemblance relation (12a) to be more degraded than a similar sentence with a Cause-Effect relation (12b). In addition, a discourse coherence-based account would predict the same asymmetry in cross-sentential ellipsis as in within-sentence ellipsis. Relations like Resemblance and Cause-Effect hold of adjacent clauses (Rohde et al. 2008), so in principle there should be no coherence-related difference between within- and cross-sentence ellipsis. Further, syntactic identity effects are observed regardless of whether the ellipsis is contained in a single sentence, or crosses a sentence boundary. This is important because, although some version of Hestvik's or Frazier and Clifton's (Arregui et al. 2006) accounts might be able to explain patterns of acceptability in Voice mismatches, these would not extend to cross-sentential ellipsis. According to the coherence account, syntactic parallelism effects appear because interpreting the Resemblance relation requires matching up arguments with parallel roles in the two VPs.

14 University of Rochester students who had not participated in either Experiment 1 or 2 participated in the experiment. 


\subsubsection{Results}

Mean $\log$ acceptability estimates for each condition are shown in Figure 3. As in Experiment 2, sentences with Voice mismatch were judged less acceptable than sentences with matching VPs, and the mismatch penalty interacted with discourse relation: degradation due to Voice mismatch was more severe when clauses were related by Resemblance. In addition, there was no interaction with ellipsis type: the discourse relation-dependent pattern of acceptability was virtually identical whether VP ellipsis was in a single sentence, or spanned two sentences.

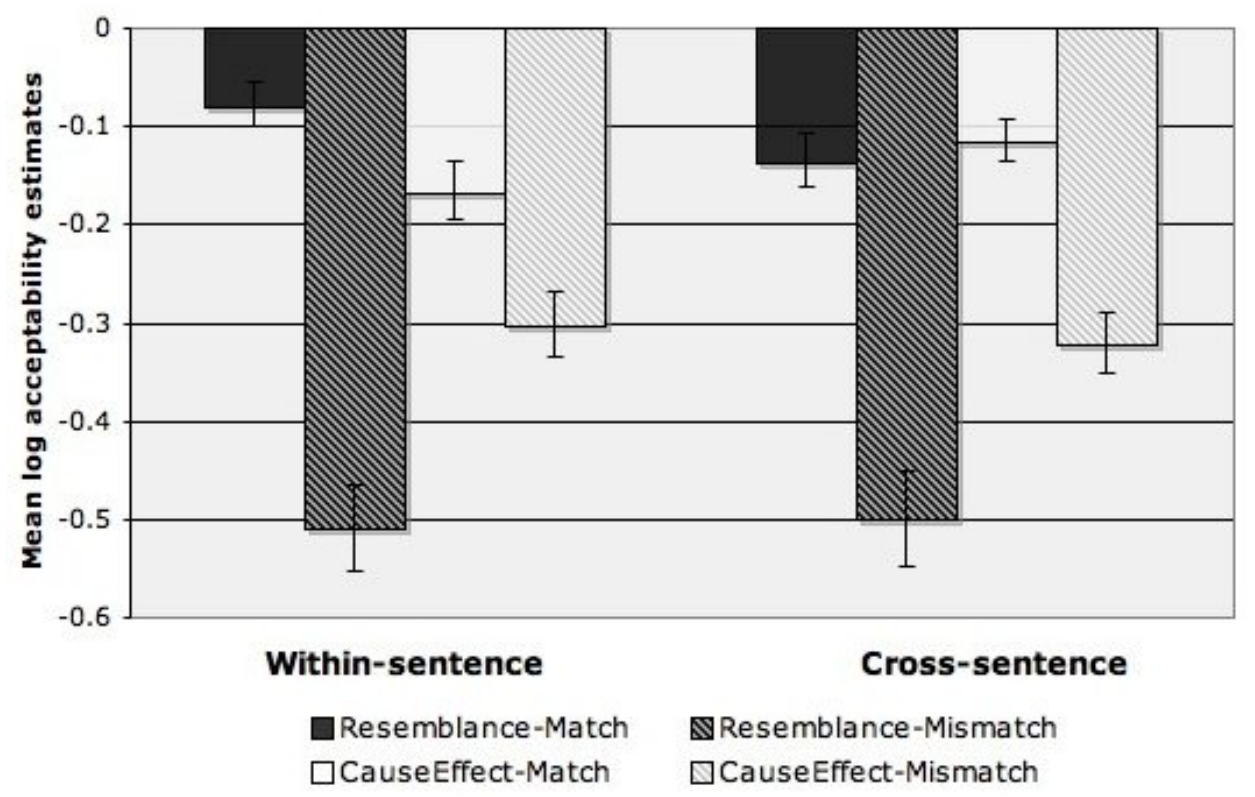

Figure 3: Experiment 3-Mean acceptability estimates (error bars represent Standard Error).

As in Experiment 2, a Cause-Effect relation between the antecedent and ellipsis clauses alleviates the degradation associated with violating syntactic identity. This is predicted by a coherence account. Further, the modulation of syntactic mismatch by discourse coherence is observed to the same extent cross-sententially as within sentences, suggesting that the same types of structural representations are accessible at the level of multiple sentence discourses as between clauses in a coordinate structure. Minimally, discourse representations must be at least structurally rich enough to encode the difference between passive and active syntax.

\section{Discussion}

Based on the results of Experiment 1, it appears that a structural account (e.g. Hestvik (1995)) can capture the distribution of strict and sloppy readings of re- 
flexives, in sentence-internal and cross-sentential VP ellipsis. Looking only at the reflexive data, it would seem that VP ellipsis resolution involves different representations when it occurs between conjoined clauses and between sentences: detailed syntactic representations of the antecedent are only accessed in the former case. However Experiments 2 and 3 suggest that the identity condition on VP ellipsis holds at a level of representation that encodes discourse coherence relations: discourse parallelism (Resemblance) enforces syntactic identity more strictly than when clauses are related causally (Cause-Effect). The gradient nature of coherence effects on syntactic mismatch suggests that the discourse representations across which VP ellipsis antecedence is established must also encode sufficiently rich structural information. One possibility would be to enrich discourse structure representations to include information about the relations among sentences, along the lines of Hardt and Romero (2004). They treat different connectives as nodes in a discourse tree, in principle allowing for a connected discourse-level representation of multiple sentences, and the hierarchical relationships among them.

The work presented here leaves certain questions open for future research. One such question is the exact nature of the structural information that VP ellipsis is sensitive to. Experiments 2 and 3 used the Voice alternation to manipulate the syntactic structure of antecedent and elided VPs, but actives and passives differ in a number of ways, including information structure (Vallduví 1992, Birner and Ward 1998) and predication structure (Williams 1980, den Dikken 2006).

There are also methodological directions suggested by the current research. One way to probe comprehenders' changing expectations is to manipulate how the discourse coherence structure is conveyed. The experiments described here rely on connectives to carry coherence information, but in actual discourse, such information is likely conveyed by a number of different cues. As an alternative to varying the connective, providing a richer preceding discourse context could bias comprehenders' expectations about the discourse relation between preceding and upcoming information. The time course (and possibly strength) of the expectations should differ by when the information about coherence structure becomes available. Using an experimental paradigm like visual world eye-tracking (Tanenhaus et al. 1995), we can track anticipatory saccades to expected upcoming referents, indicating preference for e.g. strict versus sloppy interpretations given a particular discourse context, and the timing of those expectations with respect to events in the linguistic input.

\section{References}

Arregui, Ana, Charles Clifton, Jr., Lyn Frazier, and Keir Moulton: 2006, 'Processing elided verb phrases with flawed antecedents: the recycling hypothesis', Journal of Memory and Language 55, 232-246.

Asher, Nicholas: 1993, Reference to Abstract Objects in English. Dordrecht. Asher, Nicholas, Daniel Hardt, and Joan Busquets: 2001, 'Discourse parallelism, 
ellipsis, and ambiguity', Journal of Semantics 18.

Baayen, R. Harald: 2008, Analyzing Linguistic Data: A Practical Introduction to Statistics Using R. Cambridge University Press.

Bard, Ellen Gurman, Dan Robertson, and Antonella Sorace: 1996, 'Magnitude Estimation of Linguistic Acceptability', Language 72, 32-68.

Birner, Betty J. and Gregory Ward: 1998, Information Status and Noncanonical Word Order in English. John Benjamins, Amsterdam/Philadelphia.

Chomsky, Noam: 1965, Aspects of the Theory of Syntax. MIT Press, Cambridge.

Chomsky, Noam: 1986, Barriers. MIT Press, Cambridge.

Dalrymple, Mary, Stuart M. Shieber, and Fernando C.N. Pereira: 1991, 'Ellipsis and Higher-Order Unification', Linguistics and Philosophy 14, 399-452.

den Dikken, Marcel: 2006, Relators and Linkers: The Syntax of Predication, Predicate Inversion, and Copulas. Linguistic Inquiry Monographs, MIT Press, Cambridge.

Frazier, Lyn and Charles Clifton: 2005, 'The syntax-discourse divide: processing ellipsis', Syntax 8, 121-174.

Frazier, Lyn and Charles Clifton: 2006, 'Ellipsis and Discourse Coherence', Linguistics and Philosophy 29, 319-346.

Gazdar, Gerald, Ewan Klein, Geoffrey Pullum, and Ivan Sag: 1985, Generalized Phrase Structure Grammar. Harvard University Press, Cambridge, MA.

Gelman, Andrew and Jennifer Hill: 2007, Data analysis using regression and multilevel/hierarchical models. Cambridge University Press, New York.

Hardt, Daniel and Maribel Romero: 2004, 'Ellipsis and the Structure of Discourse', Journal of Semantics 21, 375-414.

Hestvik, Arild: 1995, 'Reflexives and Ellipsis', Natural Language Semantics 3, 211-237.

Hobbs, Jerry: 1979, 'Coherence and Coreference', Cognitive Science 3, 67-90.

Johannessen, Janne Bondi: 1993, Coordination: A Minimalist Approach, Doctoral Dissertation, University of Oslo.

Katada, Fusa: 1991, 'The LF representation of anaphors', Linguistic Inquiry 287313.

Kayne, Richard: 1994, The Antisymmetry of Syntax. MIT Press, Cambridge, MA.

Kehler, Andrew: 2000, 'Coherence and the resolution of ellipsis', Linguistics and Philosophy 23, 533-575.

Kehler, Andrew: 2002, Coherence, reference and the theory of grammmar. CSLI Publications.

Kitagawa, Yoshihisa: 1991, 'Copying Identity', Natural Language and Linguistic Theory 9, 497-536.

Lebeaux, David: 1986, 'The interpretation of derived nominals', in Proceedings of CLS 22, 231-247.

Munn, Alan: 2000, 'Three types of coordination asymmetries', in K. Schwabe and N. Zhang (eds.), Ellipsis in Conjunction, 1-22. Max Niemeyer, Tubingen.

Prust, Hub, Remko Scha, and Martin van den Berg: 1994, 'A discourse perspective on verb phrase anaphora', Linguistics and Philosophy 17, 261-327.

Rohde, Hannah, Roger Levy, and Andrew Kehler: 2008, 'Implicit Causality Biases 
Influence Relative Clause Attachment'. CUNY 21 poster, University of North Carolina at Chapel Hil.

Sag, Ivan: 1976, Deletion and Logical Form, Doctoral Dissertation, MIT.

Tanenhaus, Michael, Michael Spivey-Knowlton, Kathleen Eberhard, and Julie Sedivy: 1995, 'Integration of visual and linguistic information in spoken language comprehension', Science 268, 1632-1634.

Vallduví, Enric: 1992, The Informational Component. Garland, New York.

Williams, Edwin: 1977, 'Discourse and Logical Form', Linguistic Inquiry 8, 101139.

Williams, Edwin: 1980, 'Predication', Linguistic Inquiry 11, 203-238. 